\title{
A modified high-throughput screening protocol to isolate bacteriophages from environmental samples
}

\author{
Abdallah S. Abdelsattar ${ }^{1,2}$, Rana Nofal ${ }^{1}$, Salsabil Makky ${ }^{1}$, Amera El-Sayed ${ }^{1}$, Ayman El- \\ Shibiny, ${ }^{1, *}$ \\ ${ }^{1}$ Center for Microbiology and Phage Therapy, Zewail City of Science and Technology, \\ October Gardens, 6th of October City, Giza, 12578, Egypt \\ ${ }^{2}$ Center for X-Ray and Determination of Structure of Matter, Zewail City of Science and \\ Technology, October Gardens, 6th of October, Giza, 12578, Egypt \\ *Corresponding Author aelshibiny@zewailcity.edu.eg
}

Keywords Phage Purification; phage isolation; phage characterization; jumbo bacteriophages; top agar; double agar overlay; high throughput sequencing

\begin{abstract}
In the post antimicrobial era, increasing attention is paid towards using bacteriophage (phage in short) therapy to control antibiotic-resistant bacteria. The first step in phage therapy applications is isolating highly efficient lytic phages or phage cocktails from various sources. When a double-layer- agar with around $0.7 \%$ agar in top agar is employed, it results in a low number of phage isolation with a poor resolution, and in many cases, you miss the phage. To address this problem, a low concentration of agar in top agar is examined for better phage isolation. Here, our results proved the efficiency of isolating phage upon formulating a double-layer agar with $0.3 \%$ agar in top agar. A sewage sample was collected then phages were isolated, purified, and spotted on a top layer agar with $0.3 \%$ agar. The results showed the possibility of isolating a higher number of phages on $0.3 \%$ top agar than $0.7 \%$. The finding advocates using $0.3 \%$ top agar for the double-layer agar, as it will provide fast, better, and easy phage screening and isolation.
\end{abstract}




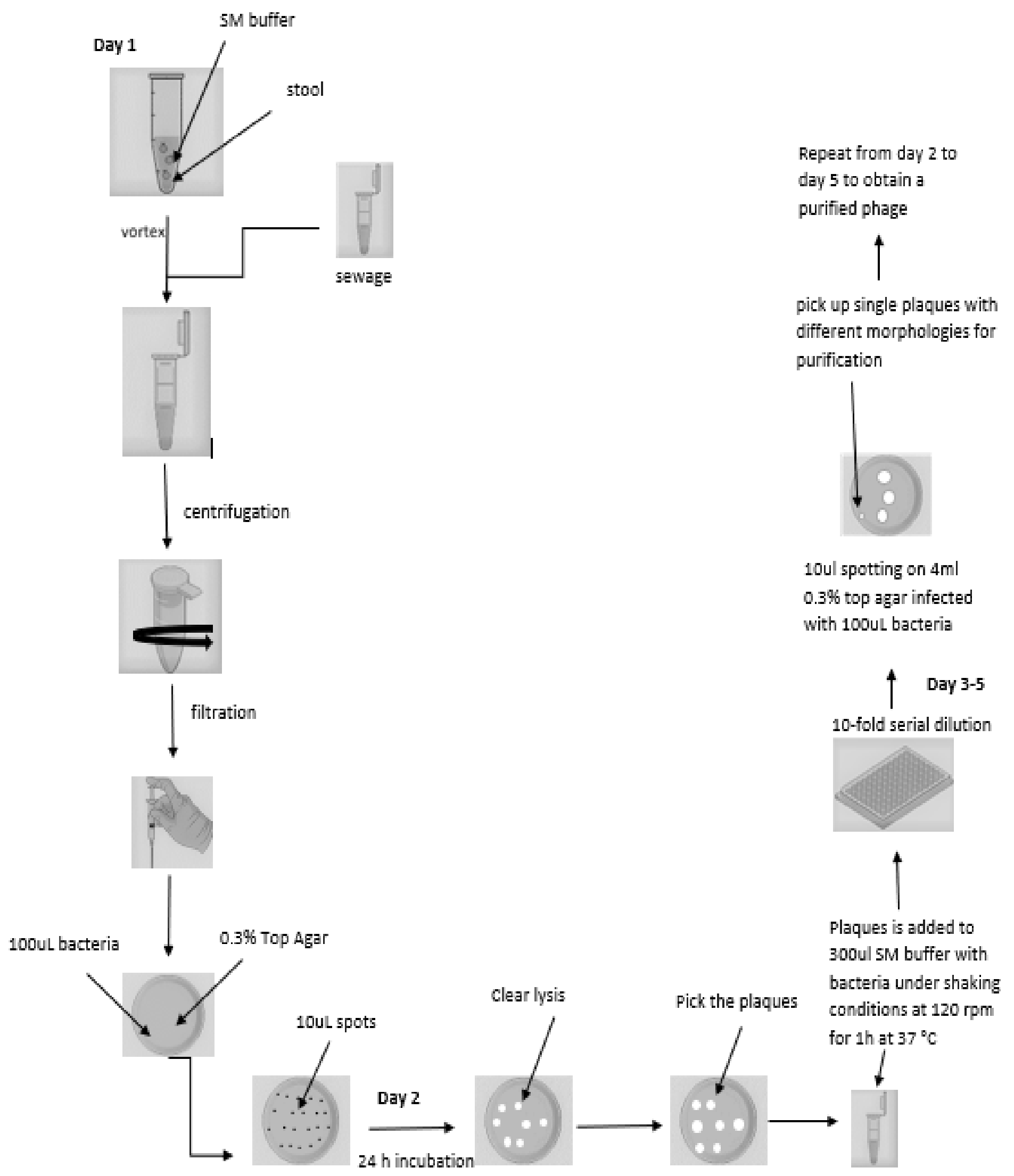

Figure 1: A graphical representation for the workflow to isolate the phage from an environmental sewage sample using a high-throughput screening method. 
Value of the Protocol
- Cost-effective, fast and simple high-throughput screening for phage isolation

- Increase the chance to obtain purified phages

- Produce a high-resolution to differentiate different morphologies of single plaques during phage purification

\section{Background information}

As phages are the natural killers of bacteria, they are found and could be isolated from the same niche of their hosts and the most common source to isolate phage is sewage water. The covenantal method of isolation relies on the using of double layers agar method. Recent studies (Echeverría-Vega et al., 2019) have reported that $0.7 \%$ of agar in top layer agar produce good quality of phage plaques, yet still it cannot help to isolate phages with relatively high molecular weight (e.g. jumbo phages) (Kamal Abdelhaleem El Jakee et al., 2008). Here, we used the standard bacteria, Escherichia coli $\mathrm{O} 157: \mathrm{H} 7($ E. coli $0157: H 7)$, as a phage host. Various concentrations of the agar $(0.7 \& 0.3 \%)$ in top agar were tested to isolate phages from collected sewage samples. To validate the work, the same environmental sample was tried on the two different agar concentrations of top agar to depict the resolution differences between the $0.3 \%$ and $0.7 \%$. Phage variations were determined based on the different morphology on the plate.

\section{Day 1: Environmental Sample Preparation and spotting}

A sewage sample was collected from the water treatment panel of Cairo, Egypt. Sample preparation is varied based on the physical state of the sewage: (A) solid sludge was first mixed with SM buffer (100 mM MgSO $4 \cdot 7 \mathrm{H}_{2} \mathrm{O} ; 10 \mathrm{mM} \mathrm{NaCl} ; 50 \mathrm{mM}$ Tris- $\mathrm{HCl} \mathrm{pH}$ 7.5) in the ratio of 1:1 (w/v) and vortexed at $1600 \mathrm{rpm}$ for $3 \mathrm{~min}$; (B) liquid sludge, which does not need the SM buffer step. Both samples were centrifuged in a cooling centrifuge at $4{ }^{\circ} \mathrm{C}$ and $8000 \mathrm{rpm}$ for $5 \mathrm{~min}$, then the resulted supernatant is filtered using $0.45 \mu \mathrm{m}$ syringe filters.

\section{N.B. Adding Chloroform, can inactivate the bacteriophages that have a lipid in the} composition of the virion (Anne K. Vidaver, R. K. Koski, 1972; Aprea et al., 2015). However, adding chloroform can stimulate the temperate phages to lysis the bacteria and obtain a high purification.

Following sample preparation and purification, the filtrate containing the phages was spotted on double-layer agar: the lower layer was prepared using TSA, while the top layer agar was prepared using TSB and $0.3 \%$ concentration of Bacto-Agar as a gelation material. A bottle of each concentration of the top agar was autoclaved, then before use, the bottle was incubated at $55{ }^{\circ} \mathrm{C}$. From this bottle, $4 \mathrm{ml}$ was withdrawn and added to $100 \mu \mathrm{l}$ of E. coli O157:H7 host strain at the exponential phase. The mixture was mixed smoothly by hand before it is added to the bottom layer of agar and left until it is solidified. Then, $10 \mu \mathrm{l}$ of around 15 filtrates containing the isolated phages were spotted on the double-layer agar. The plate was incubated for 16 hours at $37{ }^{\circ} \mathrm{C}$ under aerobic condition.

N.B. The process of enrichment by using target bacteria, in high-throughput screening, gives better results because it gives the opportunity to phages below $1000 \mathrm{PFU} / \mathrm{ml}$ (limit of 
detection) to be amplified especially. However, this process becomes highly exhaustive, costly, and time-consuming when the number of bacteria increased.

\section{Day 2: Phage Isolation using double-layer Agar}

The plaques were determined as clear zones (lysis) in the bacterial overlay as a result of phage activity. A scissor was sterilized by soaking it in $90 \%$ ethanol for few seconds before passing it on the flame. A sterile yellow tip was cut by the sterile scissor in its lower third part to facilitate the picking of plaques of each sample to be transferred into $1.5 \mathrm{ml}$ Eppendorf tube containing $900 \mathrm{ul}$ of SM buffer with the target bacteria in Log phase. in our case E. coli O157:H7, and incubated for 1 hour at $37^{\circ} \mathrm{C}$ in shaking water bath at $120 \mathrm{rpm}$.

N.B. This step is essential as it gives the chance for the phage to be eluted from the gel into the buffer and increase only the lytic phages specific for the target bacteria and let phage contamination with low concentration which will be in further steps.

The Eppendorf tube was centrifuged in a cooling centrifuge at $4{ }^{\circ} \mathrm{C}$ and $8000 \mathrm{rpm}$ for $5 \mathrm{~min}$ three times with transferring the supernatant into new sterile Eppendorf tube after each centrifugation.

N.B. the filtration step can be used after the first centrifugation instead of repeating the process of precipitation. This method will be faster but it will be costly.

The purified phages were serial diluted using a 96-well plate by filling the microplate with $180 \mathrm{uL}$ of SM buffer then adding $20 \mathrm{uL}$ of phage isolates to the first wells and mixed by withdrawing and release the mixture using micro-pipette. The tip will be discarded and replaced with another sterile one to withdraw $20 \mathrm{uL}$ of the mixture to the second wells and repeat this step to obtain 8 dilutions.

N.B. the tip should be discarded in each dilution and among different samples to avoid cross contaminations.

A $4 \mathrm{ml}$ of melted top agar at $55^{\circ} \mathrm{C}$ with a concentration of $0.3 \%$ were withdrawn from the bottle and mixed with $100 \mathrm{uL}$ of early log phase host bacteria before it is poured on the surface area of a plate containing a bottom layer of TSA. The plate was opened in a sterile area for $10 \mathrm{~min}$ or until the top agar solidified. After that $10 \mathrm{ul}$ of each phage serial dilution was spotted on the overlay from the lowest concentration to highest concentration without discarding the tip. The plate was kept open in a sterile area until the drops dried. The plates were incubated for a suitable time and temperature under aerobic conditions.

N.B. Using a multichannel pipette will reduce time and effort. The overlay may be not totally solidifying, so the plates should be handled gently and no need to be upside down.

\section{Day 3-5: Phage purification using double-layer Agar}

The single plaques is isolated from the plates based on different morphologies in the same sample or different plaques from different samples by using sterile yellow tips before adding it to an Eppendorf tube containing SM buffer. The Eppendorf tube was then centrifuged in a 
cooling centrifuge at $4{ }^{\circ} \mathrm{C}$ and $8000 \mathrm{rpm}$ for $5 \mathrm{~min}$ three times with transferring the supernatant into new sterile Eppendorf tube after each centrifugation. The purified phages is spotted on $0.3 \%$ top agar plate and incubated as described before. This step was repeated three times to ensure that you have a single phage in each eppendorf.

\section{N.B. This step is optional in the case of collecting a cocktail of phage and no need to} characterize each phage alone.

\section{Validation}

In an attempt to do a high robust screening of the phages within the environmental sample, the phage isolation is done on a low concentration of top agar using the double-layer method. Here, we used E. coli $\mathrm{O} 157: \mathrm{H} 7$ as a host to isolate phages from sewage to work against it. The results showed that, at low concentration of top agar $(0.3 \%)$, a higher resolution with a lower concentration of phage was recorded in comparison to the higher concentration of agar $(0.7 \%)$, as shown in Figure 2. The results indicated that the low concentration of the agar in the top agar will give a higher resolution to isolate the phages that could missed during isolation. This encourages the idea of isolation and the enumeration of some phages with low titer that cannot be isolated using high concentrations of agar in top agar $(0.7 \%)$.
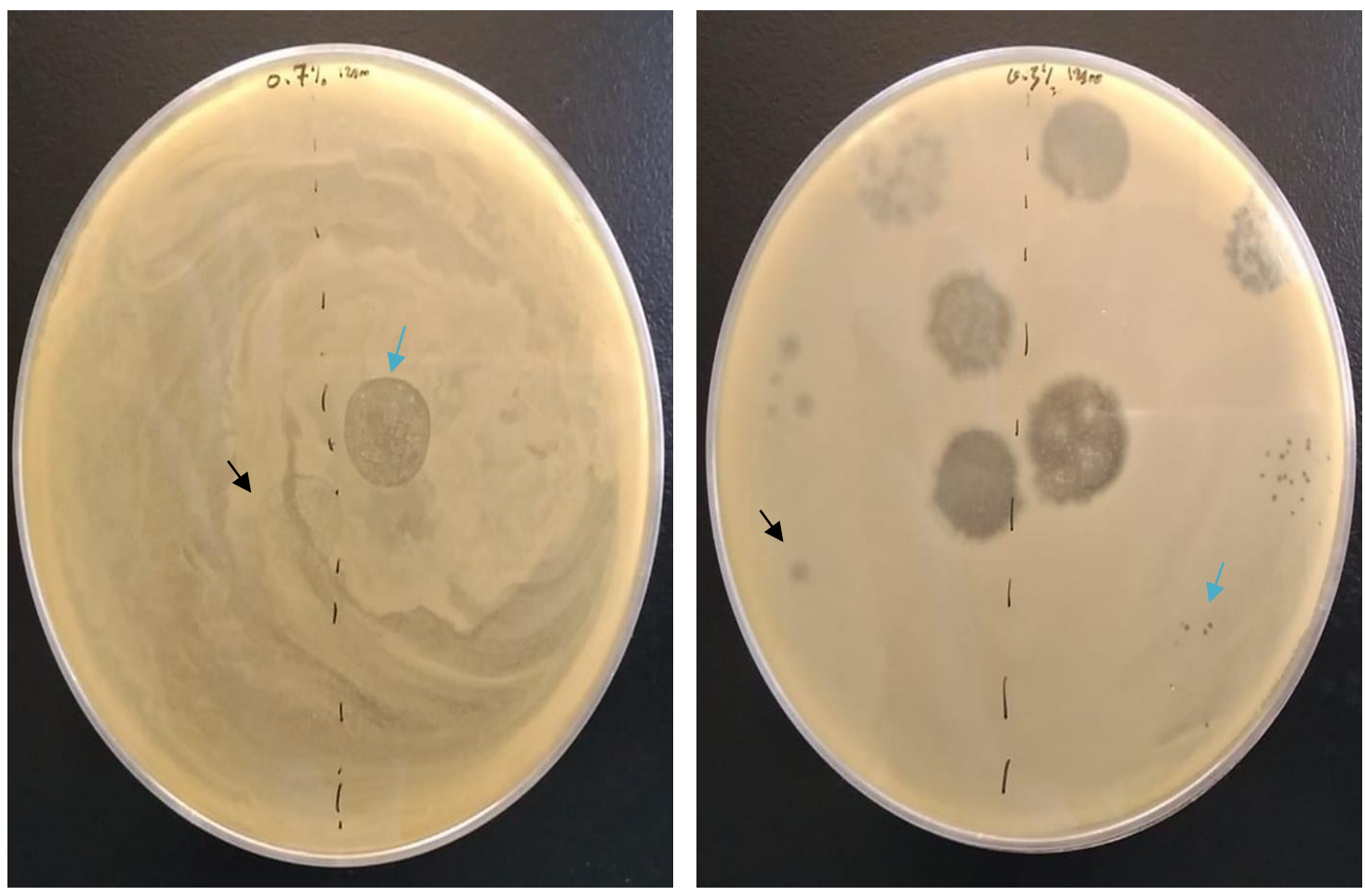

Figure 1: Phage isolation on double-layer top agar. The right plat represents the 0.3\% top agar; the left plat represents the $0.7 \%$ top agar. The dash line in both plats depicts spotting after serial dilution from two different sewage samples. The black arrows indicate sample 1 and blue arrow for sample 2. In the left plate there is a low resolution and appears only at high titer $10^{6} \mathrm{PFU} / \mathrm{ml}$. However, the right appears clearly at the level of single plaque. 


\section{Acknowledgments}

The authors would like to acknowledge the Academy of Scientific Research and Technology (ASRT), Egypt, and Zewail City of Science and Technology for financial support. This work is funded by ASRT-genetic call.

\section{References:}

ANNE K. VIDAVER, R. K. KOSKI, A. J. L. V. E. (1972). Bacteriophage $\varphi 6$ : a LipidContaining Virus of Pseudomonas phaseolicola.pdf. Journal of Virology.

Aprea, G., D’Angelo, A. R., Prencipe, V. A. ., \& Igliorati, G. (2015). Bacteriophage Morphological Characterization by Using Transmission Electron Microscopy. Journal of Life Sciences, 9(1), 214-220. https://doi.org/10.17265/1934-7391/2015.05.004

Echeverría-Vega, A., Morales-Vicencio, P., Saez-Saavedra, C., Gordillo-Fuenzalida, F., \& Araya, R. (2019). A rapid and simple protocol for the isolation of bacteriophages from coastal organisms. MethodsX, 6, 2614-2619. https://doi.org/10.1016/j.mex.2019.11.003

Kamal Abdelhaleem El Jakee, J., Sayed Ata, N., El-Jakee, J., Nagwa, A. S., Bakry, M., Zouelfakar, S. A., Elgabry, E., \& Gad El-Said, W. (2008). Characteristics of Staphylococcus aureus Strains Isolated from Human and Animal Sources Antibiotic resistance of bacterial isolates recovered from horses suffering from respiratory manifestations View project Characteristics of Staphylococcus aureus Strai. J. Agric. \& Environ. Sci, 4(2), 221-229. 